BLS 32, No 1 2006. DOI: http://dx.doi.org/10.3765/bls.v32i1.3460 (published by the Berkeley Linguistics Society and the Linguistic Society of America)

\title{
Spatial Language in Tarascan: Body Parts, Shape, and the Grammar of Location
}

\author{
MARTHA MENDOZA \\ Florida Atlantic University/University of California, Berkeley
}

\section{Introduction ${ }^{1}$}

Tarascan - also known as P'urhepecha-is a language isolate and one of the approximately 60 indigenous languages still spoken in Mexico. It is the language of the P'urhepecha people, who originally inhabited an area covering large portions of the states of Michoacán, Jalisco, Guerrero, and Guanajuato in CentralWestern Mexico. The P'urhepecha were never conquered by their enemies, the Aztecs, and developed quite an advanced civilization. Tarascan is still spoken today by about 105,000 people, or perhaps more, yet it has not been adequately investigated. To this day, only a few studies exist which deal with this most fascinating language. Tarascan speaking communities may be divided into three major areas: the Lake Pátzcuaro region, the Tarascan Plateau, and the Ravine of the Eleven Towns. The Tarascan dialect under consideration here is that of the Tarascan Plateau, or meseta tarasca, and more specifically that of the town of Angahuan, Michoacán.

Tarascan is an agglutinative type of language with an extremely rich morphology; it is also an inflectional language and has a system of cases, including the genitive, the locative, and the residential (cf. French chez). Where other languages may use adverbs, prepositions or auxiliary verbs, Tarascan employs morphological means to express meanings related to causation, direction, manner, relative orientation, repetition, voice, volition, and many others. As an example of the richness of the system, consider the following example: from the root piré 'sing' (infinitive: piréni), it is possible to derive pirépani 'to go singing', pirépuni 'to come singing', pirépunguani 'to return singing', pirénchani 'to feel like singing', pirékuekani 'to want to sing', pirépanchani 'to wish to go singing', pirépunchani 'to wish to come singing', and pirépireni 'to sing and sing, to go on singing', among numerous others.

\footnotetext{
${ }^{1}$ I would like to thank the Division of Sponsored Research at Florida Atlantic University for a grant that supported the work for this paper. Special thanks are due to my consultant, L. Gómez Bravo, for his invaluable insights into the Tarascan language.
} 


\section{Tarascan Locative Suffixes and their Meanings}

Tarascan is specially known for its abundant and complex spatial morphology. In this language, location is expressed mainly through a wide variety of body-part suffixes, which have been grammaticalized into positional-orientational markers, some of which display a high level of semantic complexity. The body-part suffixes involved include head, mouth, back, face, nose, etc. and appear in combination with verbal roots to encode varied and detailed spatial relationships between objects or the locus of affect or experience. In Tarascan there are about thirty two locative suffixes (Friedrich 1971:12)-Totonac, for example, has around thirty (Levy 1999:135); most of them possess some corporeal meaning. The suffixes may refer to a single body part such as $k$ ' $u$ 'hand', a single non-body part such as $r u$ 'road, street', or be rather complex like the extremely productive body-part suffixes that extend into several physical and psycho-social domains, such as yarhi 'face' or parha 'back'. Spatial suffixes in Tarascan constitute a formal class of bound morphemes, i.e. they do not function as independent nouns. They combine with verb roots to form more complex verbal bases; in the verbal complex, they usually appear either immediately after the root, or they appear after the root and some type of adverbial suffix (e.g. kua 'downwards', pi 'quickly', rhi 'suddenly'), if one is employed. As members of a substitution class, these suffixes are restricted to appear only one at a time in this position. After the spatial suffixes, one of several voice morphemes may be present (ku, kurhi, ra, $t a$ ), possibly followed by an adverbial suffix indicating motion, repetition, direction, and so on ( $p a, p u, m a, m u, p u n g u a, n u, n t a)$. At the end of the morphological chain, there are inflectional suffixes of various kinds (tense, aspect, person, number, and mood, and so forth) (Friedrich 1971).

In this study, I concentrate on the main corporeal suffixes, as these constitute some of the most complex and diversified of all Tarascan morphemes. Also, in the glosses to the examples, the corporeal meaning will be used for simplicity of exposition. We should note that, beyond their corporeal referents, there is an ample range of meanings that these suffixes are able to express.

In the following table, the main body-part suffixes are presented, along with their corporeal signification and, on the second column, some of the other principal meanings they can display:

Table 1. Main body-part suffixes and their meanings

1. $a$ 'stomach, intestines'

2. ch'a 'neck, throat'

3. ch'u 'buttocks, genitals'

4. $k$ ' $u$ 'hand'

5. $m u$ 'mouth, lip' central area, interior, field, yard, body of fruit or vegetable

neck of objects, narrowing, (inner) eaves

bottom of objects, underside, underneath, roofed passageway

hands of objects, tree or plant leaves

opening, orifice, edge, entrance, door, window, language, speaking 


\section{Spatial Language in Tarascan}

6. ndi 'ear, top of shoulder' ear of objects, handle, inside corner, interior surface of an angle, ground surface, branch, hearing, understanding

7. $n d u$ 'foot, leg below knee' base of plants or trees, exterior base, field

8. $\mathrm{ya} / \mathrm{ye}$ 'chest, thoracic area' interior, interior enclosure, cavity

9. yarhi 'face, eye' anterior surface of an object, interior surface, interior wall, flat (frontal) surface, flat area of the chest or of the stomach, shin, facade, emotion, mental states

10. parha 'back' posterior surface of objects, exterior surface, outside wall, belly of objects, tree trunk

11. rhu 'nose, forehead' point, tip, projection, end of object, edge, fruit, flower, seed

12. $t$ 'a 'leg, side of body' thigh, side of objects, floor, bounded flat surface 13. $t s$ ' $i$ 'head' top of objects, above, exterior upper surface

Besides these meanings, as mentioned above, some of these suffixes also participate in numerous extensions into the psycho-social domain, all of which are highly conventionalized. Due to all their extended uses, the productivity of these suffixes is truly remarkable, serving as formatives not only of verbs but of other word classes such as nouns, adverbs, and adjectives, and their combinatorial powers are extensive; as a result, they are found ubiquitously in speech and texts.

The collocational restrictions regarding combinations of verbal bases and locative suffixes are morphotactic as well as semantic and unfortunately cannot be discussed in detail here. Besides the partitive and locational meanings discussed here, body-part suffixes display intricate subtleties of meaning in combination with other morphemes, especially the voice or valence morphemes ( $t a, k u$, and $r a$ ). Most complex body-part suffixes occur before both $k u$ and $t a$ to form transitive verbs based on transitive roots; with intransitive stems ta has a causative function while $k u$ denotes state, property or location. An example that shows some of the possible combinations involving $k u$ and $t a$ and a suffix like yarhi


someone else's eyes'; mi-yarhi-ta-ni 'to close a wall' (e.g. a hole in a wall). ${ }^{2}$

\section{Application of Body-Part Suffixes to Other Entities}

Tarascan extends the use of body parts to parts of animals, objects, and locations (that stand in a relation of part to whole with objects). The human body seems to serve as the model upon which Tarascan speakers base their naming of other entities. Animals, in particular, are named as though they were humans on all fours. Also, the canonical vertical orientation and overall configuration of the human body is usually preserved in such transfers. Hence, $t s$ ' $i$ 'head' typically

\footnotetext{
${ }^{2}$ For many more examples of body-part suffixes in derivation, see especially Friedrich 1971.
} 


\section{Martha Mendoza}

names the highest area or part of an object, $c h$ 'u 'buttocks, bottom' its bottom, parha 'back' its back, and so on. However, there is no rigid imposition of this model upon a given object. For example, 'face' is not used to name any part of a pot, even though the possibility of doing so may seem to exist; rather, as discussed below, only 'back' is used. This indicates that the system is rather flexible, serving foremost the expressive needs of speakers, given that the parts of an entity do not necessarily preserve the relative location of the analogous human body part with respect to the whole or its specific shape.

As an illustration of how the system works, let us consider some examples of the use of the body-part morphemes as they are applied to an animal, such as a pig, and to different objects. For a pig, the terms used are basically the same as for a human: the pig's main parts are $t s$ ' $i$ 'head', $m u$ 'mouth', parha 'back', $a$ 'stomach', ch' $u$ 'bottom', $t$ ' $a$ 'leg', $n d u$ 'foot', and $k$ ' $u$ 'hand', all corresponding to analogous human body parts. Something to be noticed is the fact that the front legs are referred to as 'hands', which lends support to the notion that application of the suffixes is based on the overall configuration of the human body. A common object like a table has a $t s$ ' $i$ 'head' (topmost surface), ch' $u$ 'bottom' (underside), $r h u$ 'nose, forehead' (edge(s)), $t$ 'a 'leg' (each of the legs), and $n d u$ 'foot' (base of the legs). An object such as a pot has a mu 'mouth, lip' (mouth), parha 'back' (area all around the main body), $t$ ' $a$ 'side' (area on side(s) of main body), ch'u 'bottom' (bottom), and a ye/ya 'heart, chest' (interior). Here notice that parha 'back' is the suffix used for the area all around the body of the pot, where perhaps we could have expected yarhi 'face' as well; in reality, yarhi is actually used for the interior wall of the pot. Finally, a fairly featureless object such as a mat, when vertical, has a yarhi 'face' (front surface), parha 'back' (back surface), $r h u$ 'nose, forehead' (edge(s)), and ndi 'ear' (corner(s)) and, when horizontal, has a yarhi 'face' (top surface), ch'u 'bottom' (underside), rhu 'nose, forehead' (edge(s)), and $n d i$ 'ear' (corner(s)).

The assignment of part names is object-based in the sense that the orientation of the object or its position does not affect the naming of its parts. However, in the case of a fairly featureless object, such as a mat, or objects with no inherent orientation, part names are instead assigned based on the observer's perspective: yarhi 'face', for example, would have to be used to designate the flat surface facing the observer or that the observer can readily see.

\section{The Expression of Location in Tarascan}

In contrast to Western-European languages, where spatial location of objects is usually expressed by means of prepositions or case endings, Tarascan makes use of the body-part suffixes we have looked at, incorporating them into verbs. Given space constraints, I will focus mainly on the coding of location where there is contact between objects (the figure and the ground) (the case of 'the book is on the table'), rather than on the expression of location where the objects do not come into direct contact (the case of 'the tree is behind the house'), although a few remarks on this topic will be provided in a later section. 


\section{Spatial Language in Tarascan}

Through the intrinsic properties and extensive combinatorial powers of its spatial suffixes, Tarascan provides a wealth of information regarding the geometry of the entities participating in a locative event and the specific locus of the event. The choices to be made are complex and require the construal of events from an all-encompassing spatial perspective; participant entities have to be judged according to their spatial qualities, their shape, their disposition, their orientation, and, consequently, the speaker cannot remain non-committal with respect to all these variables.

For instance, we observe that the relative orientation of the object acting as 'figure' with respect to the one acting as 'ground' is obligatorily expressed in the grammatical structure, given that completely different morphemes are used according to object orientation. The function of the body part as spatial morpheme is to delimit the area of the ground where the figure can be located. Furthermore, it is absolutely obligatory to include a spatial suffix as it cannot be left out of the verbal complex. In sentences of the type 'the book is on the table,' the figure is always the subject of the sentence, and the ground is the noun marked by the locative case $r h u$. Tarascan explicitly marks the ground using the locative, unlike other Mesoamerican languages, which rely instead on the order of the two nominals involved; however, the ground may actually be left out altogether when its referent is retrievable from the context.

Consider the following sentences (in the first few examples, the verbal root is underlined, while the spatial suffix appears in bold):

\begin{tabular}{|c|c|c|}
\hline $\begin{array}{l}\text { Markadori } \\
\text { marker }\end{array}$ & 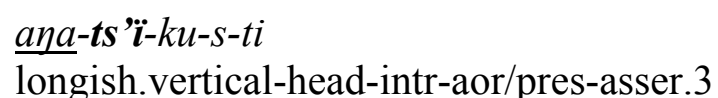 & $\begin{array}{l}\text { mesa-rhu } \\
\text { table-loc }\end{array}$ \\
\hline
\end{tabular}

(1) may be more accurately translated into English as follows: "the marker [a longish and rigid object], set in a vertical position with respect to the plane of the table [an elevated top horizontal surface], is on top [the head] of this surface." In other words, a speaker cannot fail to mention what the marker's spatial orientation is with respect to the table's surface (referred to in Tarascan as its head). ${ }^{4}$ By the same token, if the marker is not positioned in a vertical but a horizontal manner on the table, the verbal root cannot remain the same:

$\begin{array}{lll}\text { Markadori } & \text { icha-rhu-ku-s-ti} & \text { mesa-rhu } \\ \text { marker } & \text { longish.horizontal-nose-intr-aor/pres-asser.3 } & \text { table-loc }\end{array}$

'The marker is (lying) on the edge of the table.'

\footnotetext{
${ }^{3}$ Examples are not given in phonetic transcription but in ordinary Tarascan orthography, with a couple of exceptions. Note that $j=[\mathrm{h} / \mathrm{x}]$ and $x=[\check{\mathrm{s}}]$; the symbol ' after a consonant marks aspiration.

${ }^{4}$ The top surface of the table as an individuated object is referred to as kots'ikua, which also contains the suffix for head, $t s$ ' $\dddot{t}$.
} 


\section{Martha Mendoza}

(2) conveys something closer to: "the marker [a longish and rigid object], set in a horizontal position with respect to the plane of the table [a top horizontal surface], is on the edge [the nose] of this surface." In sentence (2), the body-part term has been changed from $t s ' i$ to $r h u$ to illustrate other possibilities of meaning in the language: $t s$ ' $i$ refers to the main part of the topmost surface of the table, whereas $r h u$ designates its edge(s).

Furthermore, different verbal stems are required by the grammar according to the shape of the object acting as 'figure', as exemplified by sentence (3):

$$
\begin{array}{lll}
\text { Atarakua } & \text { parha-ts'ï-ku-s-ti } & \text { mesa-rhu } \\
\text { cup } & \text { container.upright-head-intr-aor/pres-asser.3 } & \text { table-loc }
\end{array}
$$

'The cup is on the table.'

or, more accurately, "the cup [a non-round container upright], which is vertically oriented with respect to the table [a top horizontal surface], is on the top of this surface."

As can be appreciated, all the semantic complexity of shape, orientation, location, and dimension combined is coded but in a couple of morphemes.

Further examples follow:

Mikua kapa-rhu-ku-s-ti mesa-rhu cover container.upside.down-nose-intr-aor/pres-asser.3 table-loc 'The lid is upside-down on the edge of the table.'
Libru echu-ts'ï-ku-s-ti
mesa-rhu
book flat-head-intr-aor/pres-asser.3
table-loc

'The book is (lying) flat on the table.'

The location of people can also be described in a similar manner:

K'uiripu-echa (sesi) sürhi-yarhi-ku-s-ti-ksï

person-pl well row-face-intr-aor/pres-asser.3-3pl

'The people are (well) lined up on the hillside.'

(7) K'uiripu-echa icha-yarhi-ku-s-ti-ksï

person-pl longish.horizontal-face-intr-aor/pres-asser.3-3pl

'The people are lying on the hillside.'

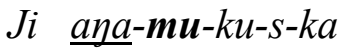
k'umanchikua-rhu
I longish.vertical-mouth-intr-aor/pres-asser.1/2 house-loc
'I am standing at the door of the house.'

In all the previous sentences, the semantic information provided in the verbal root relates to the type of object - most crucially with regard to shape - the speaker is 


\section{Spatial Language in Tarascan}

locating and to how the object is oriented, whereas the spatial suffix describes where the object is positioned. To be sure, this type of marking of spatial relationships in the grammatical structure by means of body parts does not appear to be an isolated phenomenon in Tarascan, or to be exclusive to this language. Other languages such as Totonac, Mixtec, or Zapotec behave in similar ways (cf. Brugman 1983, Brugman and Macaulay 1986, Hollenbach 1995, Levy 1999, MacLaury 1989). What is different here is the great extent to which these suffixes are integrated into the grammar of location in Tarascan and their extensive interplay with roots denoting shape, disposition, and orientation, as well as their ample use outside the human-body framework. In contrast, to give but an example, according to MacLaury (1989:135), in Zapotec there are only three body parts (namely, lip or mouth, face, and stomach) which have been extended to name locations outside the human-body framework; as shown in Table 1, in Tarascan, most body-part suffixes do so, among them ch'a 'neck', mu 'mouth', ndi 'ear', yarhi 'face, eye', parha 'back', rhu 'nose, forehead', $t$ ' $a$ 'leg, side', and $t s$ ' $i$ 'head'.

\section{The Issue of Shape}

An important consideration in Tarascan with respect to shape categories is the following: Tarascan possesses verbal roots that classify for arguments of a specific shape, which may also include an orientational or dispositional meaning component. According to Friedrich (1979:345), "the verbal roots are classificatory... in that the speaker must make decisions about the shape of their referents and the shape of the referents of the co-occurring subjects and objects."

Among the most common of these roots we find: aya 'longish, rigid object, vertically oriented' ${ }^{5}$ icha 'longish, rigid object horizontally oriented (i.e. maximal axis oriented horizontally); and, although this information is not provided in the literature, it also refers to a 3D object, such as a thick book, placed on its spine (i.e. secondary axis aligned with the vertical); echu 'flattish object horizontally oriented' (maximal axis oriented horizontally); parha '(non-round) container or hollow object upright, or facing away from the surface of contact'; kapa '(nonround) container or hollow object upside-down, or facing towards surface of contact'; kirha/irha 'round or oval object, or container with a round base'; sïrhi 'objects in a row, aligned'; chaki 'flexible object in disarray' (e.g. blankets, clothes, ropes, threads), and xakui 'elastic, stretchy object' (e.g. rubber bands, springs). Tarascan is also very rich in this aspect of its grammar, and there are many other such roots. As the glosses provided indicate, the meanings of these items can certainly get quite specific.

In the domain of numeral classifiers, historical extensions to shape are a distinct possibility. And, indeed, in Tarascan these verbal roots appear to be related to the numeral classifiers that at one point were a much more productive area of

\footnotetext{
${ }^{5}$ My semantic characterizations of these roots may differ from those of previous authors. Given the great importance of accurately characterizing these meanings, some existing definitions were refined to reflect my consultant's native speaker intuitions.
} 


\section{Martha Mendoza}

the language. In the descriptions of Tarascan made in the middle of the $16^{\text {th }}$ century (cf. Maturino Gilberti 1987 [1558]), 18 different numeral classifiers were said to be in use; these referred to shape, dimension, disposition, and other such features of objects. In the modern language, there has been a great reduction in the number and use of numeral classifiers. The only functional ones at this point are the following three: icha for long and rigid objects, echu for flat objects, and kira/irha for round ones. They are only partly obligatory in the counting of nominal expressions, as they are often missing in the noun phrase. The classifiers, if employed, are always accompanied by the nominalizer kua- and appear after the numeral, as in the following examples: ma ichakua k'erhutakua 'one finger'; t'amu irhakua enandi 'four guavas'; tembini echukua ichuskuta 'ten tortillas'. Note that in their use as nominal classifiers all three items refer to a definitional property of the objects (their shape), rather than to other more relational characteristics, such as their disposition or orientation with respect to other objects.

The combination of these shape-roots and a body-part suffix marks the existence or presence of an object of that shape at that particular location. Furthermore, by virtue of the semantics of the body-part suffix, the shape of the location is also made explicit. A few of these roots were used in sentences (1-8) above. Let us consider some more examples. Once again, the subject of the sentence represents the figure, and the noun marked by the locative case $r h u$ is the ground:

Porhechi-cha kirha-ndi-s-ti-ksï

pot-pl round-ear-aor/pres-asser.3-3pl

'The pots are on the floor.'

$\begin{array}{llll}\text { Tarheri-ri } & \text { sïndari-cha } & \text { ekua-rhu } & \text { chaki-ndi-ku-s-ti-ksï } \\ \text { farmer-gen } & \text { rope-pl } & \text { patio-loc } & \text { flexible+disarray-ear-intr- }\end{array}$

'The farmer's ropes are on the ground (in the patio).'

(11) Sïndari ma k'umanchikua-rhu chaki-ts'i-ku-s-ti-ksï

rope a house-loc flexible+disarray-head-intr-aor/presasser.3-3pl

'A rope is on the roof of the house.'

(12) Tsïtsïki ana-ye-s-ti

basu-rhu

flower longish.vertical-heart-aor/pres-asser.3 glass-loc

'The flower is (standing) inside the glass.'

Pisaroni echu-yarhi-ku-s-ti saloni-rhu

blackboard flat-face-intr-aor/pres-asser.3 classroom-loc

'The blackboard is on the classroom wall.' 


\section{Spatial Language in Tarascan}

(14) Tsikiata $\begin{aligned} & \text { parha-yarhi-ku-s-ti } \\ & \text { basket } \\ & \text { container.upright-face-intr-aor/pres-asser.3 }\end{aligned}$
The basket is (hanging) on the wall facing away from it.'

\section{Some Remarks about the Structure of Tarascan Locative Sentences}

Notice that, in all the examples we have looked at, there is no occurrence of any verb meaning 'stand', 'sit', or 'lie', etc., as it is common in other languages in locative sentences. Rather, this information is recovered from the semantics of the shape root in combination with the body part.

In addition, unlike other languages, such as Totonac or Zapotec, where body parts frequently appear outside the verb complex as part of a genitive NP construction of the type Poss-Part $+\mathrm{N}$ (e.g. its-top + house, meaning 'the roof of the house'), in Tarascan body-part morphemes never appear as independent words in the sentence. They may appear outside the verbal complex but only as formatives in derived adjectives, adverbs, or nouns; derived nouns contain a nominalizing suffix and have meanings that are related to that of the part suffix, for example, the nouns that name body parts. The following are some examples (the body-part suffix in question appears in parenthesis): chukuxukua 'elbow' (xu 'arm'), kondurhakua 'sole of the foot' ( $n d u$ 'foot'), koyekua 'chest' ( $\eta$ e 'chest'), kayarhikua 'face' (yarhi 'face'), kok'urhakua 'palm of the hand' ( $k$ 'u 'hand'), tsikat'akua 'leg' ( $t$ ' $a$ 'leg'), and so on. However, some other body part names do not seem to involve body-part suffixes: a yayastakua 'shin', jak' $i$ 'hand', $t$ 'etekua 'buttocks'.

\section{More on Shape and Location}

Levy (1999:157-8) explains that in Totonac, in some cases, the presence of the body-part suffix implies not a specific sub-area or location on the object but its overall shape. In Tarascan this reading is also possible. Such is the case in a sentence like the following:

$$
\begin{array}{ll}
\text { T'u jupa-parha-ku-sïga } & \text { enandi-ni } \\
\text { you wash-back-tran-hab+asser.1/2 } & \text { guava-obj } \\
\text { 'You wash (the body of) the guava.' } &
\end{array}
$$

This strongly implies that the action is performed on an object of a certain shape, in this specific case, an object of the roundish class. The use of another spatial suffix would mean that an object of a different shape is involved, as we see in:

$$
\begin{array}{llll}
J i & \text { jupa-yarhi-ta-sïya } & \text { mandani } & \text { uéxurhini } \\
\text { I } & \text { wash-face-tran-hab+asser. } 1 / 2 & \text { each } & \text { year } \\
\text { 'I wash the (inner) wall every year.' } & &
\end{array}
$$

The exact interpretation of such sentences is, of course, also heavily dependent on the context of the speech situation. 


\section{Martha Mendoza}

Another possibility of meaning brought about by body-part suffixes that should be mentioned is the case where the suffix refers to a part or location on the body of the subject of the sentence; in such case, the action of the verbal root is directed towards or affects the space defined by the body-part suffix. The body part represents the affected entity within a larger location. The part or area of the body may be explicitly stated as a separate noun, in which case it will be again marked by the locative $r h u$, as in the following:
Eréndira
tsïreri
$m a-k$ 'u-rha-s-ti
jak'i-rhu
Erendira corn.dough be.stuck-hand-intr-aor/pres-asser.3 hand-loc 'Erendira has corn dough stuck on her hand.'

Uanochi eni kafe jata-ka porho-ch'u-ti ${ }^{6}$ sack that coffee contain-subj have.a.hole-bottom-asser.3 'The sack that contains the coffee has a hole on the bottom.'

Ch'uru-k'u-s-ka-ni

poke-hand-aor/perf-asser.1/2-1

$$
\begin{array}{ll}
\text { ma } & \text { p'ikukua-mbo } \\
\text { a } & \text { needle-inst }
\end{array}
$$

'I poked my hand with a needle.'

$$
\begin{array}{lll}
\text { Juchiti piri-mba } & \text { aparhi-ndira-s-ti } & \text { penchumek } \\
\text { my daughter-poss.3 } & \text { burn-mouth-aor/perf-asser.3 } & \text { mouth-loc }
\end{array}
$$

'My daughter burned her mouth.'

$$
\begin{aligned}
& \text { Aparhi-ndu-s-ka-ni kondurhakua-rhu } \\
& \text { burn-feet-aor/perf-asser.1/2-1 sole.of.foot-loc } \\
& \text { 'I burned the soles of my feet.' }
\end{aligned}
$$

In the case of ambiguous suffixes (with more than one corporeal meaning), the specific location is specified by a noun marked in the locative case:
Kiti-yarhi-xa-ti eskua-rhu
rub-eyes-prog-asser. 3 eyes-loc
'He/she is rubbing his/her eyes.'

In order to indicate that the referent of a given suffix is to be found on the body of a second participant, a valence morpheme, like $k u$ or $t a$, is needed:

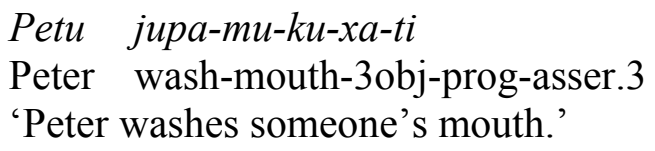

\footnotetext{
${ }^{6}$ Examples (18-22) adapted from Chamoreau (2003:276-85).
} 

I uichu katsa-ndu-ku-s-ti
tataka-ni
dem dog bite-foot-3obj-aor/perf-asser.3
'This dog bit the boy's foot.'
boy-obj

In these examples, the body part clearly constitutes the locus of affect of the verbal event. Even though the examples shown here favor human subjects, it should be evident by now that Tarascan can express all sorts of locations on both humans and objects.

Although this paper concentrates on locative events where there is direct contact between the entities involved, we need to note that these same body-part suffixes extend their domain of application metonymically from part of an object to reference to the area in space that projects out from that part, associated with that subpart, or to relative location in the space surrounding the object. Again, this is not exclusive to Tarascan but occurs in other Mesoamerican languages (Zapotec, Mixtec, Totonac). This use represents the extension of body-part suffixes as relational elements in the grammar, since the body part delimits the relative location of some object with respect to another, as exemplified in:

Libru echu-ch'u-ku-s-ti mesa-rhu book flat-bottom-intr-aor/pres-asser.3 table-loc 'The book is lying flat under the table.'
Misitu pi-t'a-ku-s-ti
porhechi-rhu cat next.to-side-intr-aor/pres-asser.3 pot-loc 'The cat is next to (the side of) the pot.'
Uaxantsïkua pi-rhu-ku-s-ti
mesa-rhu chair next.to-nose-intr-aor/pres-asser.3
table-loc
'The chair is close to the edge of the table.'

Finally, we should observe that, in Tarascan, location can also be expressed using locative adverbs and the stative verb jarháni 'to be located'. Locative adverbials are also abundant in Tarascan, among them: karhakua 'above', ketsekua 'below', maekandani 'to the side', orhepani 'in front', pexu 'behind', tatsepani 'behind', t'irek'andani 'to the right', uikixkandani 'to the left', etc. Nevertheless, body parts are at play even here, given that some of these adverbials actually contain a body-part suffix. Friedrich (1971:66) gives these examples: jandikutini (ndi 'ear') 'along the surface of an angle on a vertical axis', jandukutini ( $n d u$ 'foot') 'along the foot or base of', jarhukutini (rhu 'nose') 'along a projection'. Sentences (2830) exemplify the expression of location through locative adverbials:
(28) Tasa porhechi-rhu maekandani jarhá-s-ti cup pot-loc to.the.side be-aor/pres-asser.3 'The cup is to the side of the pot.'


Martha Mendoza

(29)



'The tree is behind the house.'

(30) Ireta juata-rhu pexu jarhá-s-ti

town mountain-loc behind be-aor/pres-asser.3

'The town is behind the mountain.'

As the number of participants in the event increases, locative sentences can get quite complex, a matter that is certainly worth of further study.

\section{Conclusion}

Tarascan is a language with no known relatives today, and its careful study should add to the body of work that seeks to provide us with a better understanding of the linguistic strategies used to talk about space and location, both cross-linguistically as well as with respect to individual languages. From this brief survey of such strategies in this particular language, we find that, just as other Mesoamerican languages, Tarascan is thoroughly preoccupied with issues of shape, orientation, and location. Further research will be needed to establish just how idiosyncratic Tarascan is with respect to spatial grammar and how this is reflected in the language. It is interesting to note, for example, the preoccupation of this language with containers and their shapes, which is consistent with the great salience and importance of such artefacts to a culture that has excelled in the domain of pottery. Moreover, we have seen that the use of locative suffixes is pervasive, and Tarascan thoroughly exploits the semantic possibilities associated with part-whole relationships; body-part suffixes have broadly diversified and have gone beyond the mere naming of parts of entities, the lexical realm, to include decidedly spatial-relational uses, in what can very well be characterized as the grammar of location. In such use of body parts, the geometry of events is emphasized and issues of shape, disposition and orientation are paramount. All in all, Tarascan constitutes an excellent example of how space has become grammar.

\section{References}

Brugman, Claudia. 1983. The Use of Body-Part Terms as Locatives in Chalcatongo Mixtec. Survey of California and Other Indian Languages 4:235-290.

Brugman, Claudia, and Monica Macaulay. 1986. Interacting Semantic Systems: Mixtec Expressions of Location. Proceedings of the Twelfth Annual Meeting of the Berkeley Linguistics Society, 315-327.

Chamoreau, Claudine. 2003. Grammaire du purépecha. Munich: Lincom Europa.

Friedrich, Paul. 1971. The Tarascan Suffixes of Locative Space. Bloomington: Indiana University Publications. 


\section{Spatial Language in Tarascan}

Friedrich, Paul. 1979. Language, Context, and the Imagination. Stanford: Stanford University Press.

Gilberti, Maturino. 1987 [1558]. Arte de la lengua de Michuacan. Morelia, Mexico: Fimax.

Hollenbach, Barbara E. 1995. Semantic and Syntactic Extensions of Body-Part Terms in Mixtecan: The Case of 'Face' and 'Foot'. International Journal of American Linguistics 61(2):168-190.

Levy, Paulette. 1999. From 'Part' to 'Shape': Incorporation in Totonac and the Issue of Classification by Verbs. International Journal of American Linguistics 65(2):127-175.

MacLaury, Robert E. 1989. Zapotec Body-Part Locatives: Prototypes and Metaphoric Extensions. International Journal of American Linguistics 55(2):119154.

Department of Languages, Linguistics, and Comparative Literature

Florida Atlantic University

777 Glades Rd.

Boca Raton, FL 33431

mmendoza@fau.edu/mmendoza@alum.berkeley.edu 